\title{
VENTILATORY STRATEGIES IN CHRONIC OBSTRUCTIVE PULMONARY DISEASE
}

\author{
B.W.P Habaragamuwa* \\ Senior Registrar in Anaesthesiology, The National Hospital, Sri Lanka \\ *Corresponding author E-mail : budhi190@yahoo.com
}

Key words: COAD, Non invasive ventilation, Oxygen therapy

Chronic obstructive pulmonary disease (COPD) is a disease characterized by air flow obstruction $\left(\mathrm{FEV}_{1}<80 \%\right.$ of predicted or $\left.\mathrm{FEV}_{1} / \mathrm{FVC}<70 \%\right)$. It is usually progressive, not completely reversible and does not change markedly over several months. The disease is predominantly caused by smoking. A small percentage can be due to alpha1 antitrypsin deficiency ${ }^{1}$.

Pathophysiologically there is airway and lung parenchymal damage leading to airway obstruction. These changes give rise to various pulmonary symptoms (cough, shortness of breath, wheezing) resulting in disability and impaired quality of life.

As anaesthetists and critical care doctors, we are involved in ventilatory management of COPD patients in following situations,

- Ventilatory support/ long term oxygen therapy in long term management of COPD patients.

- Ventilatory support in acute exacerbations in COPD patients.

- Intra operative and postoperative ventilation in patient with COPD who undergo major surgery.

\section{Long term management of COPD patients}

This consists of pharmacological and non pharmacological management. Pharmacological therapy includes bronchodilators, steroids, antibiotics, doxapram, immunization against haemophilus and pneumococcal. infection, long term oxygen therapy with or without non invasive ventilation. Non pharmacological management includes pulmonary rehabilitation and nutritional support. Lung volume reduction surgery for emphysema patients is a new management approach which has been used by many centers with good results.

Out of the treatment modalities, long term oxygen therapy (LTOT) is the only treatment demonstrated to prolong survival in control trials (Nocturnal oxygen therapy trial group. Ann Intern Med 1980; 93:391-398. Medical research council working party. Lancet 1981; 1:681-686).

\section{Indications for LTOT}

- $\mathrm{PaO}_{2}<7.3 \mathrm{KPa}$ when stable (4 weeks after an acute exacerbation).

- $\mathrm{PaO}_{2}$ 7.3 - 8.0 KPa when stable with secondary polycythaemia or nocturnal hypoxemia $\left(\mathrm{SpO}_{2}<90 \%\right.$ for $>30 \%$ of time) or peripheral oedema or pulmonary hypertension ${ }^{1}$.

Oxygen therapy (2L per minute via face mask or nasal cannula) need to be given at least 15 hours per day to get the above mentioned survival benefit. If oxygen therapy is given for more than 20 hours per day, it has been shown to reduce pulmonary vascular resistance.

In the long term management certain patients need non invasive ventilatory support other than LTOT.

\section{Clinical indications for non invasive ventilator (NIV) support in COPD}

- Symptomatic despite optimum medical treatment for COPD.

- $\mathrm{PaO}_{2}>55 \mathrm{mmHg}$.

- $\mathrm{PaO}_{2}$ - 50-54 mmHg with nocturnal desaturation $\left(\mathrm{SpO}_{2} \leq 88 \%\right.$ for more than 5 minutes while on oxygen $>2 \mathrm{~L}$ per minute).

- $\mathrm{PaO}_{2}$ - 50-54 mmHg with hospitalization related to recurrent 2 episodes in 12 months) episodes of hypercapnic respiratory failure. 
What is the rationale of NIV in the management of COPD?

It is important to know the changes happening in the COPD lung as opposed to normal lung to find the answer for the above question.

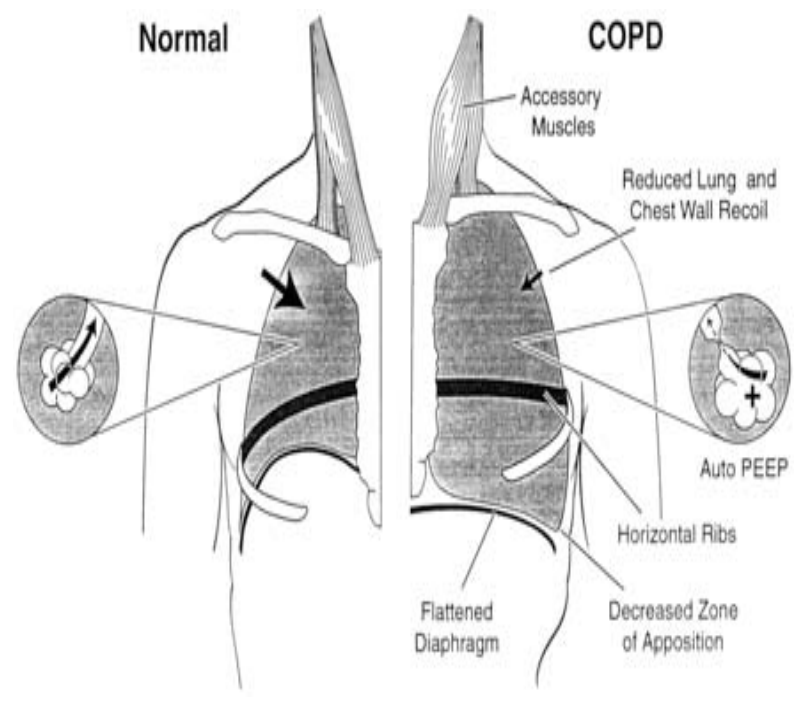

Figure 1:

Changes in COPD lung as apposed to normal lung.

- Hyperinflation in patients with emphysema has been found to place the respiratory muscles at a mechanical disadvantage.

- Flattened diaphragm shortens the sarcomere length and reduces maximal muscle force.

- Reduced bucket handle movement of the ribs impair thoracic wall compliance.

- Reduced zone of opposition between diaphragm and chest wall.

- Alveolar wall tension increase in emphysema.

This (according to Law of Laplace) leads to reduced blood supply.

- Recruitment of accessory muscles for tidal breathing.

All these changes make the energy supply demand balance unfavouarble and the end result would be chronic respiratory muscle fatigue.

NIV provide resting of the chronically fatigued muscles and this was the basis for muscle resting hypothesis. Later Fleetham J \& colleague and Catterall JR \& colleague came up with another hypothesis which was based on sleep studies done among COPD patients. They found that obstructive sleep apnea is highly prevalent among
COPD patients and obstructive sleep apnea got improved when they were given nocturnal NIV. More importantly day time gas exchange was improved in these patients.

Firstly, negative pressure ventilation was introduced to test the muscle resting hypothesis. Negative pressure ventilation was given via a wrap ventilator which was a rigid plastic or metal cage. Most of the early work had been done by Bruno and Marino which was published in Chest in 1985. They studied 16 COPD patients and found improvement in vital capacity, maximum inspiratory pressure, maximum expiratory pressure and day time $\mathrm{PaCO}_{2}$. Later studies have shown both favourable and unfavourable results with wrap ventilators. A cross over study done by the Zibrak \& colleagues did not show any benefit and they found wrap ventilator was poorly tolerated by most patients.

Nasal positive pressure ventilation (NPPV) was introduced to test the sleep hypothesis. Elliott \& colleagues and Meecham Jones \& colleagues found reduction of day time $\mathrm{PaCO}_{2}$, frequent desaturation and episodes of hypoventilation with NPPV. Later NPPV was introduced to long term follow up studies in severe COPD patients. A French trial which was a randomized controlled trial selected COPD patients with basal $\mathrm{PaCO}_{2}>50 \mathrm{mmHg}$ and randomized to two groups. One group received conventional treatment and other group received conventional treatment and NPPV. There was an improvement in survival among conventional treatment and NPPV group though it was not statistically significant. Similar findings were obtained in European multi centre trial. In conclusion, although evidence is conflicting and far from definitive, patient with severe carbon dioxide retention, particularly those with nocturnal oxygen desaturation appear most appropriate to respond favourably to NPPV.

\section{Ventilatory support in acute exacerbation of COPD}

An exacerbation is a sustained worsening of the patient's symptoms from their usual stable state which is beyond normal day to day variations, and is acute in onset.

Enhancing ventilation by unloading fatigued ventilatory muscles is an important goal in the treatment of acute exacerbations of COPD which 
is complicated by respiratory failure. Multicentre randomized controlled trials and meta analysis have shown NIV reduces numbers, reduced hospital mortality, hospital stay and accelerate symptomatic and physiological improvement ${ }^{6}$.

\section{Indications for NIV in acute COPD \\ - Worsening dyspnoea \\ - $\quad$ Acute respiratory acidosis \\ - $\quad$ Worsening oxygenation $<200$ )}

NICE guidelines also state that NIV should be considered as the treatment of choice for persisting hypercapnic respiratory failure during exacerbation of COPD despite optimal treatment. A clear plan of what to do in the event of deterioration is essential.

\section{One of the suggested treatment plans in acute COPD is as follows:}

Start optimum medical treatment.

- Provide optimum oxygen therapy - using $24 \%$

or $28 \%$ venti mask or nasal cannulae flow rate adjusted to achieve oxygen saturation $88-92 \%$.

Oxygen therapy starts from low $\mathrm{FiO}_{2}$ as these patients have hypoxic respiratory drive.

- Perform an arterial blood gas and treat as follows

- $\mathrm{pH}-<7.2$ - NIV strongly advised. Even with NIV, $50 \%$ of patients are likely to need invasive ventilation. If NIV successful, better outcome in hospital and up to 1 year.

- $\mathrm{pH}-<7.3$ - NIV strongly advised. Without NIV, $50 \%$ of patient will deteriorate until endotracheal intubation is needed or may die.

- $\mathrm{pH}-<7.35, \mathrm{RR}>23, \mathrm{PaO} 2>6 \mathrm{kPa}-\mathrm{NIV}$ advised. $80 \%$ of patient recover without NIV, but need to treat only 10 patients to avoid one intubation. Resolution of symptoms shown to be faster with NIV applied even at this stage.

Most of the studies on NIV in acute management of COPD were compared with medical treatment.

Conti G \& colleague did a randomized controlled trial comparing NIV and invasive ventilation in patients with acute respiratory failure due to COPD. They have concluded that NIV was no worse than invasive ventilation and if successful there are advantages both in the short term and in the year after hospital discharge.

\section{Indications for invasive ventilation in acute COPD \\ - $\quad$ Respiratory arrest \\ - Medical instability (hypotensive shock) \\ - Inability to protect the airway \\ - $\quad$ Excessive secretions \\ - $\quad$ Agitation / uncooperative \\ - Conditions that preclude the placement of face mask}

As clinicians, when we decide to an invasive ventilation in COPD patients, it is also important to consider the functional status of the patient (life style score), other cormobidities, age, $\mathrm{FEV}_{1}$ recording when stable, oxygen requirement when stable and body mass index. It is better to consider trial of invasive ventilation in doubtful situations.

\section{Goals in invasive ventilation in COPD}

- Improve ventilation, oxygenation, $\mathrm{CO}_{2}$ elimination and correct acidosis.

- Rest respiratory muscles while treating underlying cause.

- Prevent total inactivity of respiratory muscles.

- Avoid Dynamic pulmonary hyperinflation (DPH)

In both spontaneous and positive pressure ventilation exhalation continues till functional residual capacity. If inspiration commences before passive expiration is complete, the lung become progressively distended and gives rise to dynamic pulmonary hyperinflation.

$\mathrm{DPH}$ occurring during positive pressure ventilation is called intrinsic positive end expiratory pressure (PEEPi). Amount of PEEPi depends on lung compliance, airway tierce and respiratory variables. Some COPD patients need 20-30 seconds for full expiration and some retain up to $4 \mathrm{~L}$ above functional residual capacity in their lungs.

Intrinsic PEEP has both dynamic and static components. Experimental studies have shown dynamic PEEPi is much less than static PEEPi in patients with air flow limitation who typically have significant inhomogenity. 
Effect of PEEP on dynamic pulmonary hyperinflation in patients with airflow limitation was studied by Tan and colleague published in British journal of anaesthesia. They have studied effect of extrinsically applied PEEP (PEEPe) and PEEPi on lung volume and peak airway pressure during mechanical ventilation. They have applied PEEPe $2 \mathrm{cmH}_{2} \mathrm{O}$ increments till it exceeds PEEPi. They concluded that PEEPe may be applied cautiously at values less than PEEPi when clinically indicated, but the application of PEEPe at values greater than PEEPi may substantially aggravate lung hyperinflation.

Other studies have shown that increased inspiratory work of breathing caused by PEEPi is reduced by application of PEEPe, which may benefit during spontaneous ventilatory modes. There is little evidence to say that, matching PEEPi with PEEPe has any significant benefit in patients who are fully ventilating and not making spontaneous respiratory effort ${ }^{7}$.

\section{Adverse effects of PEEPi}

- Increase respiratory work of breathing during spontaneous ventilation.

- Reduced ability to trigger the ventilator during assisted mode of ventilation.

- Adverse haemodynamic effects of increasing intrathoracic pressure.

- Risk of barotrauma.

\section{How to counteract the DPH/PEEPi during mechanical ventilation}

- Low tidal volume.

- Lengthening expiratory time.

- Shortening inspiratory time / increase inspiratory flow.

- Reducing airway resistance

\author{
Indication for tracheostomy in COPD \\ patients \\ - Long term ventilatory support. \\ - Sputum clearance. \\ - Airway protection. \\ - Reduced dead space. \\ - Reduced airway resistance.
}

But these are not strict indications. It is important to individualize the management according to the patient's condition.

Same principals are applied when it comes to intra and post operative ventilation of a patient with COPD. Non invasive ventilation could be considered instead of continuing invasive ventilation in immediate post operative period in patients who need post operative ventilatory support. This has shown to speed up weaning.

\section{Reference}

1. Sutherland ER, Cherniack RM. Management of chronic obstructive pulmonary disease. $\mathrm{N}$ Eng $\mathrm{J}$ Med 2004;350:2689-2697.

2. Stoller JK. Acute exacerbation of COPD. N Eng J Med 2002;346:988.

3. Chronic Obstructive Pulmonary Disease. NICE (National Institute for Clinical Excellence) guidelines. $\quad 2004$. www.nice.org.uk/cgo12niceguidline

4. Josephine VL, Jadawiga AW, Mark WE, Felix SFR. Non invasive positive pressure ventilation to treat respiratory failure resulting from exacerbation of chronic obstructive pulmonary disease: Cochrane Systematic Review and Meta-analysis. BMJ Jan 2003; 326: 185.

5. Connery LE, Degnan MJ, Gujer MW, Richardson MG. Cardiovascular collapse associated with iatrogenic PEEPi in patient with obstructive airway disease. BJA 1999;83:493-495.

6. Mehta S \& colleague. Non invasive ventilation. Am J Respi Crit Care Med 2001;163:546-577.

7. Machaughton PD. New ventilators for the ICU. BJA 2006;97:57-63. 